\title{
Discrete Complex Structure on Surfel Surfaces
}

\author{
Christian Mercat \\ I3M, Université Montpellier 2 c.c. 51 \\ F-34095 Montpellier cedex 5 France \\ mercat@math . univ-montp2.fr
}

\begin{abstract}
This paper defines a theory of conformal parametrization of digital surfaces made of surfels equipped with a normal vector. The main idea is to locally project each surfel to the tangent plane, therefore deforming its aspect-ratio. It is a generalization of the theory known for polyhedral surfaces. The main difference is that the conformal ratios that appear are no longer real in general. It yields a generalization of the standard Laplacian on weighted graphs.
\end{abstract}

\section{Introduction}

Conformal parametrization of surfaces is a useful technique in image processing. The key notion is to identify the tangent plane of a surface to the field of the complex numbers in a consistent way. It allows to give a meaning to the notion of angles of two crossing paths on the surface, or equivalently to the notion of small circles around a point. A surface with such a complex structure is called a Riemann surface. A conformal, holomorphic or analytic function between two Riemann surfaces is a function that preserves angles and small circles.

This notion has been tremendously successful in mathematics and engineering; an aim of this paper is to define its discrete counterpart in the context of the surfel surfaces, defining for example discrete polynomials (see Fig. 6). It is a crucial notion in texture mapping for example: consider a particular animal skin that is known to contain small patterns of a given shape, like round disks; if this texture is rendered in a way that stretches these patterns into ovals, the picture will be wrongly interpreted by the viewer, the distortion being understood as conveying an information of tilt of the underlying surface.

The technique has many other uses, like vector fields on surfaces, surface remeshing, surface recognition or surface interpolation. One of its main features is its rigidity: the Riemann mapping theorem tells you that a surface topologically equivalent to a disc can be conformally mapped to the unit disc, uniquely up to the choice of three points. In this way, surfaces which are very different can be mapped to the same space where their features can be compared. There is much less freedom than in the case of harmonic mapping for example (kernel of the Laplacian, see (4)), which depends on many arbitrary choices, which are too numerous or too sensitive in many cases. This technique is surprisingly robust to changes in the bulk of the surface and the dependency on the boundary conditions can be relaxed as well [1, putting rigidity where the data is meaningful.

D. Coeurjolly et al. (Eds.): DGCI 2008, LNCS 4992, pp. 153-164, 2008.

(C) Springer-Verlag Berlin Heidelberg 2008 
This technique has been widely used in the polyhedral surfaces community [1, 2, 3, 4, 5, 6, 7, In this paper we will describe its adaptation to the case of digital surfaces made of surfels, square boundaries of voxels in $\mathbf{Z}^{3}$ that constitute a simple combinatorial surface where each edgel belongs to one or two surfels. We develop in this article the theory and algorithms needed for an actual implementation on computers of these notions to the context of surfel surfaces.

The additional information that we use in order to give the digital surface a conformal structure is the data of the normal direction [8, 9, 10,

In the first section 3. we will present how this information encodes a non real discrete conformal structure. Then, in Sec. 4 we will recall elements of de Rham cohomology and apply them in Sec. 5 to define a discrete Hodge star from this conformal structure. How this leads to a theory of discrete Riemann surfaces will be explained in Sec. 6.77, first recalling the real case, then generalizing to the complex case, which is the main technical result of this paper.

\section{Previous Work}

Conformal maps are present in a lot of areas. They are called analytic, holomorphic, meromorphic or monodriffic. They are related to harmonic maps because they are in particular complex harmonic. In the context of discrete geometry processing, they were used mainly by the polyhedral community, for example Desbrun and al. in [1,2, or Gu and Yau 4, 5, 6,7, Circle packings have been used as well to approximate conformal maps, see [3] and references therein.

\section{Conformal Structure}

We show here how a surfel surface equipped with normals defines a discrete conformal structure and gives a geometric interpretation to holomorphic maps.

A discrete object is a set of points in $\mathbf{Z}^{3}$, each center of its Voronoi cell called voxel. A voxel is a cube of unit side, its six faces are called surfels. A digital surface $\Sigma$ made of surfels is a connected set of surfels. We will restrict ourselves to surfaces such that every edge in $\Sigma$ belongs to at most two surfels [11. The edges that belong to only one surfel are called boundary edges. Let us call (the indices stand for dimensions) $\left(\nabla_{0}, \nabla_{1}, \diamond_{2}\right)$ the sets of vertices, edges and surfels of this cellular decomposition $\diamond$ of the surface $\Sigma$.

Note that this cellular decomposition is bipartite: there exists a bicoloring of its vertices, that can be colored whether black or white, no adjacent vertices have the same color. We consider the surfels diagonals, their end points share the same color, forming dual black and white diagonals.

We call $\Gamma$ the 1-skeleton graph, whose vertices $\Gamma_{0}$ are given by the black vertices and its edges $\Gamma_{1}$ by the black diagonals. It can be completed into a cellular decomposition of the surface by adding faces $\Gamma_{2}$ for each white vertex. Similarly we define its Poincaré dual $\Gamma^{*}$ composed of the white vertices, white diagonals and faces associated with black vertices. We will refer to $\Gamma$ as the primal 


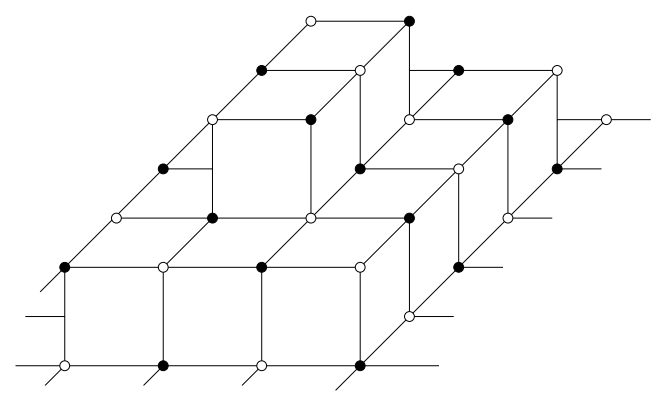

Fig. 1. A surfel surface

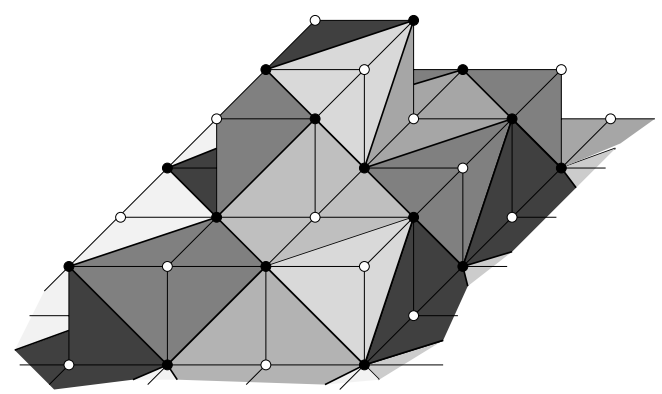

Fig. 2. The cellular decomposition $\Gamma$ associated with black vertices

(black) graph and to $\Gamma^{*}$ as its dual (white) graph. The $k$-cells of $\Gamma$ are nothing else than the $2-k$ cells of $\Gamma^{*}: \Gamma_{k} \approx \Gamma_{2-k}^{*}$.

The data of a normal direction at each surfel is a broadly used feature of digital surfaces $8,9,10$. This normal might come from a digital scanner, or be computed from the digital surface itself by various means on which we won't elaborate. These consistent normals give an orientation to the surface.

This normal is used to project a given surfel comprising the four vertices $\left(x, y, x^{\prime}, y^{\prime}\right)$ to the local tangent plane. This projection deforms the square into a parallelogram such as in Fig. 3. Its diagonals are sent to segments which are no longer orthogonal in general. We identify the tangent plane with the complex plane, up to the choice of a similitude. We call $Z$ this local map from the cellular decomposition to the complex numbers. Each diagonal $\left(x, x^{\prime}\right)$ and $\left(y, y^{\prime}\right)$ is now seen as a complex number $Z\left(x^{\prime}\right)-Z(x)$, resp. $Z\left(y^{\prime}\right)-Z(y)$.

For example we can project the standard digital plane of cubes associated with $P_{0}: x+y+z=0$ onto this (constant tangent) plane $P_{0}$ and get the rhombi pattern appearing in Fig. 4

We then associate to each diagonal $\left(x, x^{\prime}\right) \in \Gamma_{1}$ the (possibly infinite) complex ratio $i \rho$ of the dual diagonal by the primal diagonal, as complex numbers.

$$
i \rho\left(x, x^{\prime}\right):=\frac{Z\left(y^{\prime}\right)-Z(y)}{Z\left(x^{\prime}\right)-Z(x)}
$$




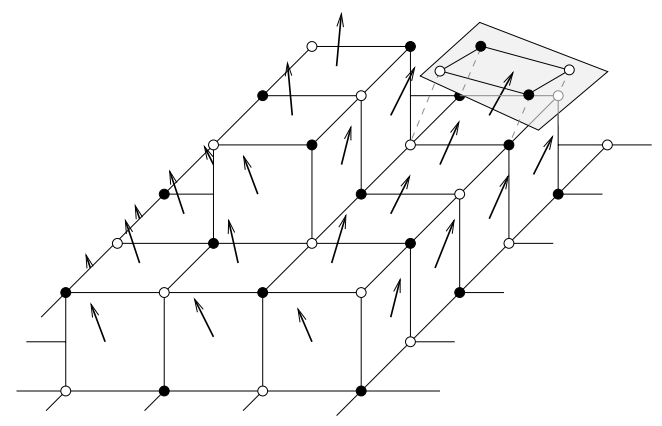

Fig. 3. A surfel projected onto the local tangent plane

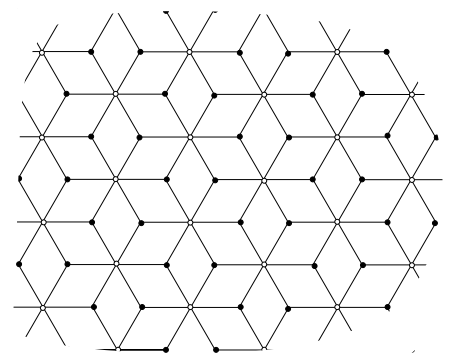

Fig. 4. The digital plane $x+y+z=0$ projected. Note that $\Gamma$ is the hexagonal lattice, $\Gamma^{*}$ its triangular dual.

This ratio clearly does not depend on the choice of identification between the tangent plane and the field of complex numbers.

We will prefer the number $\rho$ to the ratio $i \rho$ and still call it abusively the ratio. This number does not depend on the orientation of the edge, for $\rho\left(x, x^{\prime}\right)=$ $\rho\left(x^{\prime}, x\right)$. The number $\rho$ is real whenever the normal is orthogonal to (at least) one of the diagonals, that is to say, when the two diagonals are orthogonal when projected to the tangent plane. We will call this eventuality the real case. It is so, for example, in the standard plane case in Fig. 4 where its value is the constant $\rho_{\text {hex }}=\tan \left(\frac{\pi}{6}\right)=1 / \sqrt{3}$. The flat square grid $\mathbb{Z}^{2}$ is associated with the constant 1. Large or small values away from 1 appear whenever the surfels are flattened away from the square aspect ratio. The complex valuess appear when the surfel is slanted away from the orthogonal conformation.

We call this data of a graph $\Gamma$, whose edges are equipped with a complex number $\rho$, a discrete conformal structure, or a discrete Riemann surface.

We equip the dual edge $\left(y, y^{\prime}\right) \in \Gamma_{1}^{*}$ of the complex constant $\rho\left(e^{*}\right)=1 / \rho(e)$. In the example Fig. 4, its value is the constant $\rho_{\text {tri }}=\tan \left(\frac{\pi}{3}\right)=\sqrt{3}=1 / \rho_{\text {hex }}$.

We define a map $f: \diamond_{0} \rightarrow \mathbb{C}$ as discrete holomorphic with respect to $\rho$ if and only if, it respects the ratio for each surfel:

$$
\forall\left(x, y, x^{\prime}, y^{\prime}\right) \in \diamond_{2}, f\left(y^{\prime}\right)-f(y)=i \rho\left(x, x^{\prime}\right)\left(f\left(x^{\prime}\right)-f(x)\right) .
$$




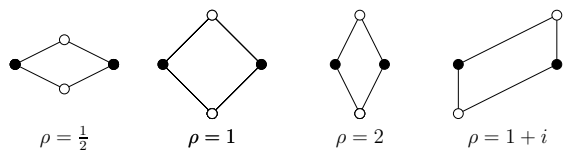

Fig. 5. Several surfel conformations and the associated ratio $\rho$

In the continuous complex analytic theory, a holomorphic function $f$ is a complex function from the complex plane to itself, which is complex differentiable; that is to say, it is recognized by the fact that its action on a neighborhood around a point $z_{0} \in \mathbf{C}$ is locally a similitude $z \mapsto a z+b$ where $b=f\left(z_{0}\right)$ and $a=f^{\prime}\left(z_{0}\right)$, the derivative of $f$ at $z_{0}$ with respect to the complex variable $z$. It sends little circles to little circles.

In the same fashion, a discrete conformal map sends each quadrilateral surfel to another quadrilateral whose diagonals have the same ratio $\rho$ and it can be pictured as sending polygons that form the double $\Lambda$ into similar polygons that still fit together. For example, the hexagonal and triangular lattices, hidden in Fig. 4, can actually be drawn on the same picture by simply joining the middles of the edgels together. The hexagons and triangles are simply both shrunk by a factor half and fit together at their vertices. A discrete conformal map is recognizable in the sense that it sends each of these regular hexagons and equilateral triangles to polygons of the same shape, touching with the same combinatorics. The discrete derivative is encoded in how much each polygon has been inflated or shrunk, and rotated.
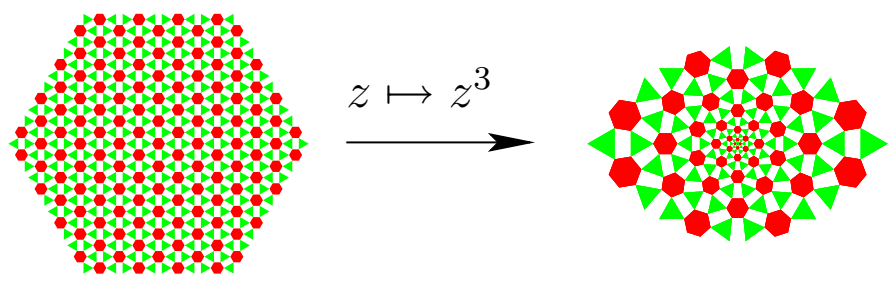

Fig. 6. The discrete version of the map $z \mapsto z^{3}$ in the hexagonal/triangular case

\section{De Rham Cohomology}

In the continuous theory of surfaces, the notion of complex structure relies on the existence of an operator on 1 -forms such that $*^{2}=-\mathrm{Id}$, called the Hodge star. In orthonormal local coordinates $(x, y)$, it is defined on 1 -forms by $*(f d x+g d y)=$ $-g d x+f d y$. The discrete analogous of these local coordinates are given by these pairs of dual diagonals $\left(x, x^{\prime}\right)$ and $\left(y, y^{\prime}\right)$.

In order to follow further this analogy, we first have to define the spaces of discrete functions and discrete forms such as $d x, d y$. This is done in the theory of de-Rham cohomology 12 . 
In this section we recall elements of de Rham cohomology, with functions, boundary operator and forms, in which the notions of discrete analytic functions take place. The novelty is that we need to double everything to get the best out of the complex structure.

We define $\Lambda:=\Gamma \sqcup \Gamma^{*}$, disjoint union of the two dual graphs, that we will call the double graph. Its vertices $\Lambda_{0}=\diamond_{0}$ are the same as the vertices of the surfel cellular decomposition $\diamond$, its edges $\Lambda_{1}$ are the black and white diagonals, its faces $\Lambda_{2}$ is a set in bijection with its vertices. This doubling can look artificial at first sight but is in fact very useful in practice, allowing for nicer formulae because $\Lambda$ is self-dual by construction. We could as well define complex functions as being real on the vertices $\Gamma_{0}$ and pure imaginary on the faces $\Gamma_{2}$, but it is not as practical. The case is similar to the continuous where functions and 2 -forms are essentially the same set but treated differently.

The complex of chains $C(\Lambda)=C_{0}(\Lambda) \oplus C_{1}(\Lambda) \oplus C_{2}(\Lambda)$ is the vector space (over $\mathbb{R}$ the field of reals) spanned by vertices, edges and faces. It is equipped with a boundary operator $\partial: C_{k}(\Lambda) \rightarrow C_{k-1}(\Lambda)$, null on vertices and fulfilling $\partial^{2}=0$. The kernel ker $\partial=: Z_{\bullet}(\Lambda)$ of the boundary operator are the closed chains or cycles. The image of the boundary operator are the exact chains. The homology of $\Lambda$ is the space of cycles modulo exact chains. It encapsulates all there is to know about the topology of the surface.

The dual spaces of forms are called cochains, denoted with upper indices for the dimension, are functions from chains to the field of complex numbers. $C^{k}(\Lambda):=\operatorname{Hom}\left(C_{k}(\Lambda), \mathbb{C}\right)$. The space $C^{0}(\Lambda)$ of 0 -forms is the linear span of functions of the vertices, 1 -forms are functions of the oriented edges, 2 -forms are functions of the faces. Coupling is denoted by functional and integral notation: the value of a 1 -form $\alpha$ evaluated on an edge $\left(x, x^{\prime}\right) \in \Lambda_{1}$ will be denoted by $\int_{x}^{x^{\prime}} \alpha$, similarly, $f(x)$ for a 0 -form on a vertex, $\iint_{F} \omega$ for a 2 -form on a face. The dual of the boundary operator is called the coboundary operator $d: C^{k}(\Lambda) \rightarrow C^{k+1}(\Lambda)$, defined by Stokes formula:

$$
\int_{x}^{x^{\prime}} d f:=f\left(\partial\left(x, x^{\prime}\right)\right)=f\left(x^{\prime}\right)-f(x), \quad \iint_{F} d \alpha:=\oint_{\partial F} \alpha,
$$

where $\oint$ denotes the circulation of a 1-form along a closed contour. A cocycle is a closed cochain and we note $\alpha \in Z^{k}(\Lambda)$. The cohomology of $\Lambda$ is the space of cocycles modulo the exact forms.

We define an exterior wedge product, denoted $\wedge$, for 1-forms living either on edges $\nabla_{1}$ or on their diagonals $\Lambda_{1}$, as a 2 -form living on surfels $\nabla_{2}$. The formula for the latter is:

$$
\iint_{\left(x, y, x^{\prime}, y^{\prime}\right)} \alpha \wedge \beta:=\frac{1}{2}\left(\int_{\left(x, x^{\prime}\right)} \alpha \int_{\left(y, y^{\prime}\right)} \beta-\int_{\left(y, y^{\prime}\right)} \alpha \int_{\left(x, x^{\prime}\right)} \beta\right)
$$


The exterior derivative $d$ is, as it should be, a derivation for the wedge product, for functions $f, g$ and a 1 -form $\alpha$ :

$$
d(f g)=f d g+g d f, \quad d(f \alpha)=d f \wedge \alpha+f d \alpha .
$$

\section{Hodge Star}

After having set the spaces where our operator is going to act, we show, in this section, how the discrete conformal structure allows to define a Hodge star, that is to say, an operator verifying $*^{2}=-\mathrm{Id}$ on 1-forms. It breaks forms into holomorphic and anti-holomorphic parts. Moreover, we show that this decomposition is robust to flips and that parallel planes have isomorphic decompositions.

Definition 1. The Hodge star is defined on functions and 2-forms by

$$
\begin{aligned}
& *: C^{k}(\Lambda) \rightarrow C^{2-k}(\Lambda) \\
& C^{0}(\Lambda) \ni f \mapsto * f: \iint_{F} * f:=f\left(F^{*}\right), \\
& C^{2}(\Lambda) \ni \omega \mapsto * \omega:(* \omega)(x):=\iint_{x^{*}} \omega,
\end{aligned}
$$

and on 1 -forms, on the dual edges $\left(y, y^{\prime}\right)=\left(x, x^{\prime}\right)^{*} \in \Lambda_{1}$, given the (complex) discrete conformal structure $\rho\left(x, x^{\prime}\right)=r e^{i \theta}$, by

$$
\left(\begin{array}{l}
\int_{x}^{x^{\prime}} * \alpha \\
\int_{y}^{y^{\prime}} * \alpha
\end{array}\right):=\frac{1}{\cos \theta}\left(\begin{array}{cc}
-\sin \theta & -\frac{1}{r} \\
r & \sin \theta
\end{array}\right)\left(\begin{array}{l}
\int_{\left(x, x^{\prime}\right)} \alpha \\
\int_{\left(y, y^{\prime}\right)} \alpha
\end{array}\right) .
$$

Notice that the Hodge star is a real transformation. The fact that it is well defined relies on the fact that two dual diagonals are associated with inverse numbers: $\rho\left(y, y^{\prime}\right)=1 / \rho\left(x, x^{\prime}\right)=\frac{1}{r} e^{-i \theta}$ (using the former notation). It fulfills $*^{2}=$ Id for functions and 2-forms, and $*^{2}=-$ Id for 1 -forms:

We have, on the surfel $\left(x, y, x^{\prime}, y^{\prime}\right) \in \diamond_{2}$,

$$
\frac{1}{\cos ^{2} \theta}\left(\begin{array}{cc}
-\sin \theta & -\frac{1}{r} \\
r & \sin \theta
\end{array}\right)^{2}=\frac{1}{\cos ^{2} \theta}\left(\begin{array}{cc}
\sin ^{2} \theta-1 & 0 \\
0 & \sin ^{2} \theta-1
\end{array}\right)=-I_{2} .
$$

Because of this property, we can define it on the complexified forms and functions. It breaks the complex 1-forms into two eigenspaces associated to eigenvalues $-i$ and $+i$ called respectively type $(1,0)$ and $(0,1)$ forms.

Definition 2. A holomorphic form $\alpha \in C_{\mathbf{C}}^{1}(\Lambda)$ is a closed type (1,0) form, that is to say, $\alpha$ is such that $d \alpha=0$ and $* \alpha=-i \alpha$. An anti-holomorphic form is a closed type $(0,1)$ form.

A function $f$ is holomorphic iff its exterior derivative $d f$ is as well holomorphic, that is to say, on a surfel $\left(x, y, x^{\prime}, y^{\prime}\right) \in \diamond_{2}, f\left(y^{\prime}\right)-f(y)=i \rho\left(x, x^{\prime}\right)\left(f\left(x^{\prime}\right)-\right.$ $f(x))$.

$A$ meromorphic form with a pole at $F \in \diamond_{2}$, is a type $(1,0)$ form, closed except on the surfel $F$. Its lack of closeness is called its residue at $F$. 
Holomorphic and meromorphic functions are tremendously important in mathematics, they are behind all the keys on a calculator like polynomials, inversion, cosine, tangent, exponential, logarithm... This article defines the framework in which their discrete counterparts take place.

An interesting feature of the theory is its robustness with respect to local moves. A discrete holomorphic map defined on a discrete Riemann surface is mapped by a canonical isomorphism to the space of discrete holomorphic maps defined on another discrete Riemann surface linked to the original one by a series of flips. These flips are called in the context of discrete conformal structures electrical moves. They come in three kinds, the third one being the flip, the others being irrelevant to our context.
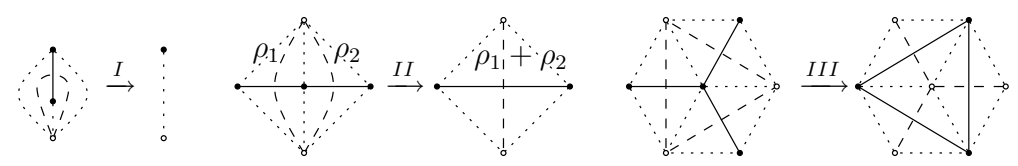

Fig. 7. The electrical moves

The third move corresponds to the flip, it is called the star-triangle transformation. To three surfels (drawn as dotted lines in Fig. 7) arranged in a hexagon, whose diagonals form a triangle of conformal parameters $\rho_{1}, \rho_{2}$ and $\rho_{3}$, one associates a configuration of three other surfels whose diagonals form a three branched star with conformal parameters $\rho_{i}^{\prime}$ (on the opposite side of $\rho_{i}$ ) verifying

$$
\rho_{i} \rho_{i}^{\prime}=\rho_{1} \rho_{2}+\rho_{2} \rho_{3}+\rho_{3} \rho_{1}=\frac{\rho_{1}^{\prime} \rho_{2}^{\prime} \rho_{3}^{\prime}}{\rho_{1}^{\prime}+\rho_{2}^{\prime}+\rho_{3}^{\prime}} .
$$

The value of a holomorphic function at the center of an hexagon is overdetermined with respect to the six values on the hexagon. These values have to fulfill a compatibility condition, which are the same for both hexagons, therefore a holomorphic function defined on a discrete Riemann surface can be uniquely extended to another surface differing only by a flip 13 .

This means in particular that a discrete holomorphic function defined on the standard plane in Fig. 4 can be followed through all its other parallel deformations and is not sensitive to some added noise (flips deleting or inserting extra voxels) provided the normal vector is unchanged with respect to the discrete plane value: the space of holomorphic functions on these parallel or noisy planes are in one-to-one correspondence. This theoretical robustness has yet to be experimentally observed in practice because the normal vectors are not independent and a noisy plane will have noisy normal vectors as well. This will be the subject of a forthcoming article.

For real conformal structures, the formulae are simpler, we present them independently before generalizing them to the complex case. 


\section{The Real Case}

We present in this section the formulae in the real case, for the Hodge star, the Laplacian, the scalar product and the different energies, Dirichlet and conformal.

When the complex structure is defined by a real ratio $\rho$, for example in the hexagonal/triangular standard discrete plane case, then, for each surfel, the Hodge star takes the simpler form

$$
\begin{aligned}
& *: C^{k}(\Lambda) \rightarrow C^{2-k}(\Lambda) \\
& C^{0}(\Lambda) \ni f \mapsto * f: \iint_{F} * f:=f\left(F^{*}\right), \\
& C^{1}(\Lambda) \ni \alpha \mapsto * \alpha: \int_{e} * \alpha:=-\rho\left(e^{*}\right) \int_{e^{*}} \alpha, \\
& C^{2}(\Lambda) \ni \omega \mapsto * \omega:(* \omega)(x):=\iint_{x^{*}} \omega .
\end{aligned}
$$

The endomorphism $\Delta:=-d * d *-* d * d$ is, in this real case, the usual discrete Laplacian: Its formula on a function at a vertex $x \in \Gamma_{0}$ with neighbours $x_{1}, \ldots, x_{V} \in \Gamma_{0}$ is the usual weighted averaged difference:

$$
(\Delta(f))(x)=\sum_{k=1}^{V} \rho\left(x, x_{k}\right)\left(f(x)-f\left(x_{k}\right)\right) .
$$

The space of harmonic forms is defined as its kernel.

Together with the Hodge star, they give rise, in the compact case, to the usual weighted scalar product on 1-forms:

$$
(\alpha, \beta):=\iint_{\diamond_{2}} \alpha \wedge * \bar{\beta}=(* \alpha, * \beta)=\overline{(\beta, \alpha)}=\frac{1}{2} \sum_{e \in \Lambda_{1}} \rho(e) \int_{e} \alpha \int_{e} \bar{\beta}
$$

The $\ell^{2}$ norm of the 1-form $d f$, called the Dirichlet energy of the function $f$, is the average of the usual Dirichlet energies on each independant graph

$$
\begin{aligned}
E_{D}(f):=\|d f\|^{2} & =(d f, d f)=\frac{1}{2} \sum_{\left(x, x^{\prime}\right) \in \Lambda_{1}} \rho\left(x, x^{\prime}\right)\left|f\left(x^{\prime}\right)-f(x)\right|^{2} \\
& =\frac{E_{D}\left(\left.f\right|_{\Gamma}\right)+E_{D}\left(\left.f\right|_{\Gamma^{*}}\right)}{2} .
\end{aligned}
$$

The conformal energy of a map measures its conformality defect, relating these two harmonic functions. A conformal map fulfills the Cauchy-Riemann equation

$$
* d f=-i d f .
$$

Therefore a quadratic energy whose null functions are the holomorphic ones is

$$
E_{C}(f):=\frac{1}{2}\|d f-i * d f\|^{2} .
$$


It is related to the Dirichlet energy through the same formula as in the continuous case:

$$
\begin{aligned}
E_{C}(f) & =\frac{1}{2}(d f-i * d f, d f-i * d f) \\
& =\frac{1}{2}\|d f\|^{2}+\frac{1}{2}\|-i * d f\|^{2}+\operatorname{Re}(d f,-i * d f) \\
& =\|d f\|^{2}+\operatorname{Im} \iint_{\diamond_{2}} d f \wedge \overline{d f} \\
& =E_{D}(f)-2 \mathcal{A}(f)
\end{aligned}
$$

where the area of the image of the application $f$ in the complex plane has the same formula

$$
\mathcal{A}(f)=\frac{i}{2} \iint_{\diamond_{2}} d f \wedge \overline{d f}
$$

as in the continuous case. For a face $\left(x, y, x^{\prime}, y^{\prime}\right) \in \diamond_{2}$, the algebraic area of the oriented quadrilateral $\left(f(x), f\left(x^{\prime}\right), f(y), f\left(y^{\prime}\right)\right)$ is given by

$$
\begin{aligned}
\iint_{\left(x, y, x^{\prime}, y^{\prime}\right)} d f \wedge \overline{d f} & =i \operatorname{Im}\left(\left(f\left(x^{\prime}\right)-f(x)\right) \overline{\left(f\left(y^{\prime}\right)-f(y)\right)}\right) \\
& =-2 i \mathcal{A}\left(f(x), f\left(x^{\prime}\right), f(y), f\left(y^{\prime}\right)\right) .
\end{aligned}
$$

When a holomorphic reference map $z: \Lambda_{0} \rightarrow \mathbb{C}$ is chosen, a holomorphic (resp. anti-holomorphic) 1-form $d f$ is, locally on each pair of dual diagonals, proportional to $d z$, resp. $d \bar{z}$, so that the decomposition of the exterior derivative into holomorphic and anti-holomorphic parts yields $d f \wedge \overline{d f}=\left(|\partial f|^{2}+|\bar{\partial} f|^{2}\right) d z \wedge$ $d \bar{z}$ where the derivatives naturally live on faces.

All these concepts turn into an actual machinery that can be implemented on computer and a full featured theory mimicking the theory of Riemann surfaces to a surprisingly far extent 14. In particular in the rhombic case, the notion of polynomials and exponentials, differential equations, logarithm (called the Green function) follow through. A striking difference is the fact that constants are of dimension two, the constants on the graph $\Gamma$ being independent from the constants on the dual graph $\Gamma^{*}$.

\section{Non Real Conformal Structure}

In this section we give formulae for the scalar product, the Dirichlet energy and the Laplacian in the complex case. Nothing more is needed in order to implement computation of global conformal parametrization of surfel surfaces.

In the complex case, the dual graphs, which are independent in the real case, are no longer independent and are mixed together.

The first construction that needs an adaptation is the scalar product. The formula (5) still defines a positive definite scalar product which is preserved by $*$, even though the last equality must be replaced by a mixed sum over the two dual edges:

$$
(\alpha, \beta)=\frac{1}{2} \sum_{e \in \Lambda_{1}} \frac{\int_{e} \alpha}{\operatorname{Re}(\rho(e))}\left(|\rho(e)|^{2} \int_{e} \bar{\beta}+\operatorname{Im}(\rho(e)) \int_{e^{*}} \bar{\beta}\right) .
$$


The Dirichlet energy mixes the two dual graphs as well:

$$
E_{D}(f):=\|d f\|^{2}=\frac{1}{2} \sum_{e \in \Lambda_{1}} \frac{\left|f\left(x^{\prime}\right)-f(x)\right|^{2}}{\operatorname{Re}(\rho(e))}\left(|\rho(e)|^{2}+\operatorname{Im}(\rho(e)) \frac{\overline{f\left(y^{\prime}\right)-f(y)}}{\overline{f\left(x^{\prime}\right)-f(x)}}\right)
$$

and the Laplacian no longer splits on the two independent dual graphs: Given $x_{0} \in \Lambda_{0}$, with dual face $x_{0}^{*}=\left(y_{1}, y_{2}, \ldots, y_{V}\right) \in \Lambda_{2}$ and neighbours $x_{1}, x_{2}, \ldots, x_{V}$

$\in \Lambda_{0}$, with dual edges $\left(x_{0}, x_{k}\right)^{*}=\left(y_{k}, y_{k+1}\right) \in \Lambda_{1}$, and $y_{V+1}=y_{1}$, we have

$$
\Delta(f)\left(x_{0}\right)=\sum_{k=1}^{V} \frac{1}{\operatorname{Re}(\rho(e))}\left(|\rho(e)|^{2}\left(f\left(x_{k}\right)-f(x)\right)+\operatorname{Im}(\rho(e))\left(f\left(y_{k+1}\right)-f\left(y_{k}\right)\right)\right) .
$$

This Laplacian is still real and involves not only the neighbors along the diagonals of surfels on a weighted graph, which is the star used for the usual Laplacian, but all the vertices of the surfels that contains the vertex.

\section{Conclusions and Acknowledgments}

This paper defines the theory of analytic functions on digital surfaces made of surfels. It required more than a mere adaptation of the theory known in the polyhedral community since the conformal parameters are in general not real.

In forthcoming papers, this theory, already put to use in the context of polyhedral surfaces, will be implemented in the context of surfel surfaces: The first applications that come to mind are the recognition of digital surfaces, simple ones and of higher topology through the computation of their discrete period matrices [15], the analysis of vector fields on digital surfaces allowed by the Hodge theorem that decomposes vector fields into rotational and divergence free parts, the creation of vector fields with given circulation properties, in general the correct discrete treatment of partial differential equations on a digital surface which are solved by analytic functions in the continuous case, like incompressible fluid dynamics for example.

The author would like to thank the referees for useful comments, the forthcoming Géométrie Discrète ANR project, in particular Valérie Berthé (LIRMM) and Rémy Malgouyres (LLAIC) for discussions that brought the main idea that made this paper possible.

\section{References}

1. Desbrun, M., Meyer, M., Alliez, P.: Intrinsic parameterizations of surface meshes. Computer Graphics Forum 21, 209-218 (2002)

2. Desbrun, M., Kanso, E., Tong, Y.: Discrete differential forms for computational modeling. In: SIGGRAPH 2006: ACM SIGGRAPH 2006 Courses, pp. 39-54. ACM Press, New York (2006) 
3. Kharevych, L., Springborn, B., Schröder, P.: Discrete conformal mappings via circle patterns. ACM Trans. Graph. 25(2), 412-438 (2006)

4. Gu, X., Yau, S.: Computing conformal structures of surfaces. Communications in Information and Systems 2(2), 121-146 (2002)

5. Gu, X., Yau, S.: Surface classification using conformal structures. International Conference on Computer Vision (2003)

6. Gu, X., Yau, S.-T.: Global conformal surface parameterization. In: SGP 2003: Proceedings of the 2003 Eurographics/ACM SIGGRAPH symposium on Geometry processing. Eurographics Association, Aire-la-Ville, Switzerland, pp. 127-137 (2003)

7. Jin, M., Wang, Y., Yau, S.-T., Gu, X.: Optimal global conformal surface parameterization. In: VIS 2004: Proceedings of the conference on Visualization 2004, pp. 267-274. IEEE Computer Society Press, Washington, DC, USA (2004)

8. Lenoir, A., Malgouyres, R., Revenu, M.: Fast computation of the normal vector field of the surface of a 3-D discrete object. In: Miguet, S., Ubéda, S., Montanvert, A. (eds.) DGCI 1996. LNCS, vol. 1176, pp. 101-112. Springer, Heidelberg (1996)

9. Lenoir, A.: Fast estimation of mean curvature on the surface of a $3 \mathrm{~d}$ discrete object. In: Ahronovitz, E. (ed.) DGCI 1997. LNCS, vol. 1347, pp. 175-186. Springer, Heidelberg (1997)

10. Malgouyres, R.: A discrete radiosity method. In: Braquelaire, A., Lachaud, J.-O., Vialard, A. (eds.) DGCI 2002. LNCS, vol. 2301, pp. 428-438. Springer, Heidelberg (2002)

11. Malgouyres, R., Burguet, J.: Strong Thinning and Polyhedrization of the Surface of a Voxel Object. Discrete Appl. Math. 125(1) (2003). In: Nyström, I., Sanniti di Baja, G., Borgefors, G. (eds.) DGCI 2000. LNCS, vol. 1953, pp. 93-114. Springer, Heidelberg (2000)

12. Farkas, H.M., Kra, I.: Riemann surfaces, 2nd edn. Springer-Verlag, New York (1992)

13. Bobenko, A.I., Mercat, C., Suris, Y.B.: Linear and nonlinear theories of discrete analytic functions. Integrable structure and isomonodromic Green's function. J. Reine Angew. Math. 583, 117-161 (2005)

14. Mercat, C.: Discrete Riemann surfaces and the Ising model. Comm. Math. Phys. 218(1), 177-216 (2001)

15. Mercat, C.: Discrete riemann surfaces. In: Papadopoulos, A. (ed.) Handbook of Teichmüller Theory, vol. I. IRMA Lect. Math. Theor. Phys., Eur. Math. Soc., Zürich, vol. 11, pp. 541-575 (2007) 